\title{
Image Registration using the Shearlet Transform
}

\author{
Glenn R. Easley ${ }^{a}$, Monica Barbu-McInnis ${ }^{a}$, Demetrio Labate $^{b}$, \\ a MITRE \\ ${ }^{b}$ Department of Mathematics, University of Houston
}

\begin{abstract}
In this paper, we present an algorithm for image registration utilizing the shearlet representation. The shearlet framework allows one to collect multi-scale and multi-directional feature information from multidimensional data that can be used to create key feature vectors that are scale, rotation, and shift invariant. These key feature vectors produce a transformation that will align the sensed image to the source image. We demonstrate our registration algorithm on various medical databases.
\end{abstract}

Keywords: shearlets, wavelets, image registration, feature detection, feature matching

\section{INTRODUCTION}

Image registration aims to align two or more images of the same scene taken at different times, from different viewpoints and/or by different sensors. Such image processing task is a crucial step in a wide range of imaging problems, including medical imaging and remote sensing. In remote sensing, for example, standard applications of image registration include environmental monitoring and change detection where valuable information needs to be combined from multiple images. In medical imaging, applications include combining CT and MRI data to obtain a more complete understanding of the disease at a particular time or during longitudinal studies.

Due to its relevance and wide applicability, the problem of image registration has been investigated in multiple studies and using a variety of techniques, which are often highly dependent on the specific application. The point of this paper is not to provide a comprehensive overview on image registration; we refer the readers to Ref.s 1-6 for such synopsis. Instead, we will focus on a particular group of classical and effective methods for image registration in order to show how they can be improved by introducing some emerging ideas from multiscale analysis and sparse representations. For simplicity, in this paper we will only consider the problem in the 2-dimensional setting.

\subsection{Image registration methodology}

We will focus on the classical problem where two images of the same scene are acquired from different viewpoints and we aim to gain a larger view of the scanned scene. Depending on the classes of images (e.g., iris, brain, arial scans) to be registered, on the possible types of image degradation (e.g., frequency attenuation, atmospheric distortion) and the types of geometric deformation between the images (e.g., rigid, projective, curved), different methods have been proposed. Nevertheless, most registration methods include of the following four steps. ${ }^{6}$

- Feature detection. Its goal is to detect salient and distinctive objects in an image, e.g., edges, contours, line intersections, corners.

- Feature matching. Its goal is to establish a correspondence between the features detected in the sensed image and those detected in the reference image.

- Transform model estimation. Its goal is to estimate the parameters of the function mapping sensed image to referenced image.

- Image resampling and transformation. The sensed image is transformed by means of the mapping functions, and this may require to re-sample the image by interpolating its values at non-integer coordinates.

G.R.E: E-mail: geasley@mitre.org, M.B.M: E-mail:monicabm@mitre.org, D.L: E-mail: dlabate@math.uh.edu 
Hence, the first step in most image registration methods involves the extraction of features in images. Such features may include region or line features that are detected by means of segmentation methods. Object contours or high variance points and corners have also been used as features in the registration process (cf. Ref.6 for examples to such methods). Once features have been detected in the reference and sensed images, the next step is to establish the correspondence between the features. Feature matching may be obtained by using the pairwise spatial relation of features. An alternative and frequently more reliable approach to match features is to use their invariant (or almost invariant) descriptors. Ideally, such descriptors should be not only invariant (i.e., the descriptions of the corresponding features from the reference and sensed image have to be the same) but also stable, that is, the description of a slightly deformed feature should be close to the description of the original feature. The current state-of-the-art on local descriptors includes the SIFT descriptor ${ }^{7}$ (Scale Invariant Feature Transform), which computes the gradient vector for each pixel in the feature point's neighborhood and builds a histogram of gradient directions; the GLOH descriptor ${ }^{8}$ (Gradient Location and Orientation Histogram), which is an extension of the SIFT descriptor designed to increase the robustness and distinctiveness of the descriptors; the SURF descriptor ${ }^{9}$ (Speeded Up Robust Features), which is partly inspired by the SIFT descriptor but employs Haar wavelet filters to increase robustness and decrease computation time.

The detection of features and selection of informative and robust descriptors is the most critical process of the registration algorithm, as it determines the ability to capture the relevant image structure. Once this step is completed, the parameters of the best transformation that models the mapping function between the reference and sensed image can then be estimated using standard robust methods such as LMedS (Least Median of Square) and RANSAC (Random Sampling Consensus). Finally, the sensed image is transformed by means of the mapping functions (with possible re-sampling).

The main contribution of this paper is the introduction of an innovative approach to extract local image descriptors based on the shearlet transform, a multiscale approach combining multiresolution analysis and high directional selectivity. Using this idea, we build a class of invariant and robust descriptors that can be seen as a refinement of the SURF descriptors and that perform very competitively when applied for image registration tasks.

The rest of the paper is organized as follows. Section 2 presents the shearlet representation, which is critical in our method for feature extraction, and Section 3 provides a brief overview of the scattering transform, which we use to obtain invariant and stable image descriptors. The construction of the shearlet extracted features is presented in Section 4 and the results of our method are discussed in Section 5. Concluding remarks are in Section 6 .

\section{SHEARLETS}

Shearlets emerged during the last decade as a powerful refinement of conventional wavelets and other classical multiscale representations. ${ }^{10,11}$ Similar to curvelets, ${ }^{12}$ shearlets are well-localized waveforms defined not only over a range of scales and locations, like wavelets, but also over multiple orientations and with highly anisotropic shapes. As a result, they are especially efficient to capture edges, corners and other significant geometric features in images.

Roughly speaking, in dimension $n=2$, shearlets are generated by the action of anisotropic dilations and shear transformations on an appropriate set of generators $\psi^{(\nu)} \in L^{2}\left(\mathbb{R}^{2}\right)$, that is,

$$
\psi_{j, \ell, k}^{(\nu)}(x)=2^{3 j / 2} \psi^{(\nu)}\left(B_{\nu}^{\ell} A_{\nu}^{j} x-k\right),
$$

for $j \geq 0,-2^{j} \leq \ell \leq 2^{j}, k \in \mathbb{Z}^{2}, \nu=1,2$, where $A_{1}=\left(\begin{array}{ll}4 & 0 \\ 0 & 2\end{array}\right), A_{2}=\left(\begin{array}{ll}2 & 0 \\ 0 & 4\end{array}\right)$ are the anisotropic dilation matrices and $B_{1}=\left(\begin{array}{ll}1 & 1 \\ 0 & 1\end{array}\right), B_{2}=B_{1}^{t}$ are the shear matrices. The indices $j, \ell, k$ are associated with a range of scales, orientations and locations, respectively.

Shearlet properties are well explained by illustrating the shearlet construction in the Fourier domain. ${ }^{13}$ 


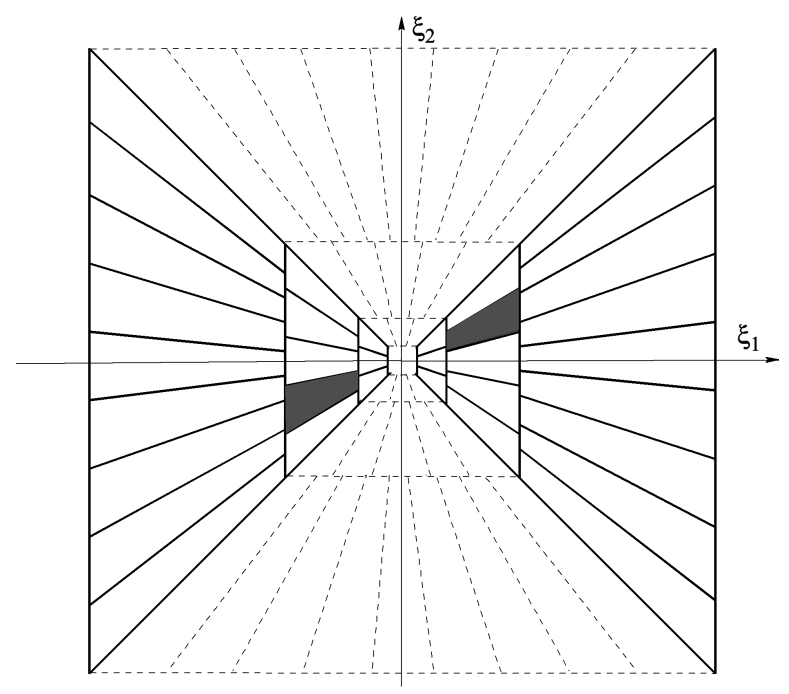

Figure 1. Tiling of the Fourier plane associated with the Parseval frame of shearlets $(n=2)$. The Fourier support $\Sigma_{j, \ell}$ of a typical shearlet element is shown as a solid grey region. The horizontal and vertical cones are partitioned into directional subbands, visualized by solid and and dashed lines, respectively.

Let $\phi \in C^{\infty}([0,1])$ be a 'bump' function with $\operatorname{supp} \phi \subset\left[-\frac{1}{8}, \frac{1}{8}\right]$ and $\phi=1$ on $\left[-\frac{1}{16}, \frac{1}{16}\right]$. For $\xi=\left(\xi_{1}, \xi_{2}\right) \in \mathbb{R}^{2}$, let $\Phi(\xi)=\Phi\left(\xi_{1}, \xi_{2}\right)=\phi\left(\xi_{1}\right) \phi\left(\xi_{2}\right)$ and define

$$
W(\xi)=W\left(\xi_{1}, \xi_{2}\right)=\sqrt{\Phi^{2}\left(2^{-2} \xi_{1}, 2^{-2} \xi_{2}\right)-\Phi^{2}\left(\xi_{1}, \xi_{2}\right)}
$$

The functions $W_{j}^{2}=W^{2}\left(2^{-2 j}\right.$.), $j \geq 0$, have support inside the Cartesian coronae

$$
C_{j}=\left[-2^{2 j-1}, 2^{2 j-1}\right]^{2} \backslash\left[-2^{2 j-4}, 2^{2 j-4}\right]^{2}
$$

and produce a smooth tiling of the frequency plane:

$$
\Phi^{2}\left(\xi_{1}, \xi_{2}\right)+\sum_{j \geq 0} W^{2}\left(2^{-2 j} \xi_{1}, 2^{-2 j} \xi_{2}\right)=1 \text { for }\left(\xi_{1}, \xi_{2}\right) \in \mathbb{R}^{2}
$$

To obtain an angular partition, let $V \in C^{\infty}(\mathbb{R})$ so that $\operatorname{supp} V \subset[-1,1], V(0)=1$,

$$
|V(u-1)|^{2}+|V(u)|^{2}+|V(u+1)|^{2}=1 \quad \text { for }|u| \leq 1 .
$$

Hence, the 'fine-scale' shearlets are the functions

$$
\hat{\psi}_{j, \ell, k}^{(\nu)}(\xi)=2^{-3 j / 2} W\left(2^{-j} \xi\right) F_{\nu}\left(\xi A_{\nu}^{-j} B_{\nu}^{-\ell}\right) e^{2 \pi i \xi A_{\nu}^{-j} B_{\nu}^{-\ell} k},
$$

where $F_{1}\left(\xi_{1}, \xi_{2}\right)=V\left(\frac{\xi_{2}}{\xi_{1}}\right)$ and $F_{2}\left(\xi_{1}, \xi_{2}\right)=V\left(\frac{\xi_{1}}{\xi_{2}}\right)$ and the matrices $A_{\nu}, B_{\nu}$ are as above. As shown in Ref. 13, the functions (2) can be (essentially) written in space-domain as (1).

We remark that the functions $\hat{\psi}_{j, \ell, k}^{(1)}$ can be written as

$$
\hat{\psi}_{j, \ell, k}^{(1)}(\xi)=2^{-2 j} W\left(2^{-2 j} \xi\right) V\left(2^{j} \frac{\xi_{2}}{\xi_{1}}-\ell\right) e^{2 \pi i \xi A_{1}^{-j} B_{1}^{-\ell} k},
$$

showing that their supports are contained inside the trapezoidal regions

$$
\Sigma_{j, \ell}=\left\{\left(\xi_{1}, \xi_{2}\right): 2^{2 j-4}<\left|\xi_{1}\right|<2^{2 j-1},\left|\frac{\xi_{2}}{\xi_{1}}-\ell 2^{-j}\right| \leq 2^{-j}\right\}
$$


within the horizontal cone $\left|\xi_{2}\right| \leq\left|\xi_{1}\right|$ of the Fourier plane. Similar properties hold for the functions $\hat{\psi}_{j, \ell, k}^{(2)}$, whose supports are contained within the vertical cone $\left|\xi_{2}\right|>\left|\xi_{1}\right|$ of the Fourier plane. The tiling of the Fourier plane associated with the shearlet construction is shown in Fig. 1.

A smooth Parseval frame for $L^{2}\left(\mathbb{R}^{2}\right)$ is obtained by combining the 'fine-scale' shearlets together with a coarse scale system, associated with the low frequency region.* That is, we define a shearlet system for $L^{2}\left(\mathbb{R}^{2}\right)$ as

$$
\left\{\widetilde{\psi}_{-1, k}: k \in \mathbb{Z}^{2}\right\} \cup\left\{\widetilde{\psi}_{j, \ell, k, \nu}: j \geq 0,|\ell|<2^{j}, k \in \mathbb{Z}^{2}, \nu=1,2\right\}
$$

where $\widetilde{\psi}_{-1, k}=\check{\Phi}(\cdot-k)$ and $\widetilde{\psi}_{j, \ell, k, \nu}=\psi_{j, \ell, k}^{(\nu)}$. With compact notation, we denote this system as

$$
\left\{\widetilde{\psi}_{\mu}, \mu \in M\right\},
$$

where $M=M_{C} \cup M_{F}$ are the indices associated with coarse-scale and fine-scale shearlets, respectively; that is, $\left.M_{C}=\left\{(j, k): j=-1, k \in \mathbb{Z}^{2}\right\}, M_{F}=\left\{(j, \ell, k, \nu): j \geq 0,|\ell|<2^{j}, k \in \mathbb{Z}^{2}, \nu=1,2\right)\right\}$. We have the following result from Ref. 13.

TheOREm 2.1. The system of shearlets (3) is a Parseval frame for $L^{2}\left(\mathbb{R}^{2}\right)$. That is, for any $f \in L^{2}\left(\mathbb{R}^{2}\right)$, we have the reproducing formula

$$
f=\sum_{\mu \in M}\left\langle f, \widetilde{\psi}_{\mu}\right\rangle \widetilde{\psi}_{\mu}
$$

with convergence in the $L^{2}$-norm. All elements $\left\{\widetilde{\psi}_{\mu}, \mu \in M\right\}$ are $C^{\infty}$ and compactly supported in the Fourier domain.

By combining multiscale analysis and high directional sensitivity, shearlets can provide highly sparse representations for a large class of multidimensional data, outperforming conventional multiscale representations. In particular, for cartoon-like functions ${ }^{\dagger}$, a simplified model of images with edges, they are (nearly) optimally sparse. $^{14}$

THEOREM 2.2. Let $f \in E^{2}$, the class of carton-like functions in $\mathbb{R}^{2}$, and $f_{N}$ be its $N$-term approximation obtained by taking the $N$ largest coefficients in the shearlet representation of $f$. Then:

$$
\left\|f-f_{N}\right\|_{2}^{2} \leq C N^{-2}(\log N)^{3} .
$$

Ignoring the log factor, this result yields the optimal decay rate (no other basis or frame can achieve faster decay rate than $\mathrm{N}^{-2}$ ) and it outperforms, in particular, wavelet approximations whose error rate is only of order $N^{-1}$. We remark that curvelets achieve the same type of approximation rate as shearlets. ${ }^{12}$

The mapping of a function $f \in L^{2}\left(\mathbb{R}^{2}\right)$ into the elements $\left\{\left\langle f, \widetilde{\psi}_{\mu}\right\rangle\right\}$ is called the 2D shearlet transform of $f$. Hence, the shearlet transform maps a function into a set of coefficients associated with a range of scales, orientations and locations.

\section{SCATTERING TRANSFORM}

Scattering representations can be powerful discriminators for texture and classification. ${ }^{15}$ This is due to their invariance to translation and small deformations, which is achieved by building a representation from wavelet coefficients using the modulus and average pooling functions.

To formalize their construction, let $G$ be a group of rotation $r$ of angles $2 k \pi / 2$ for $0 \leq k<2$ (the general case is formulated in terms of $K$ angles but for our purposes we only need two directions). A wavelet system is obtained by rotating a single band-pass filter $\psi$ by $r$ and dilating it by $2^{j}$ for $j \in \mathbb{Z}$ so that

$$
\psi_{\lambda}(u)=2^{-2 j} \psi\left(2^{-j} r^{-1} u\right) \text { with } \lambda=2^{-j} r .
$$

${ }^{*}$ To ensure that all elements of this combined shearlet system are $C_{c}^{\infty}$ in the Fourier domain, the elements whose supports overlap the boundaries of the cone regions in the frequency domain are slightly modified. ${ }^{13}$

${ }^{\dagger}$ Roughly speaking, this is the class of functions that are $C^{2}$ regular away from $C^{2}$ edges. ${ }^{14}$ 
In particular, the wavelet transform of $f$ is defined as $\left\{f * \psi_{\lambda}(u)\right\}_{\lambda}$. Let $U[\lambda] f=\left|f * \psi_{\lambda}\right|$. Given a sequence $p=\left(\lambda_{1}, \lambda_{2}, \ldots, \lambda_{m}\right)$ describing a path, we define an ordered product of operators as

$$
U[p] f=U\left[\lambda_{m}\right] \cdots U\left[\lambda_{2}\right] U\left[\lambda_{1}\right] f
$$

with $U[0] f=f$. A scattering transform along the path $p$ is then defined by

$$
\bar{S} f(p)=\mu_{p}^{-1} \int U[p] f(u) d u
$$

where $\mu_{p}=\int U[p] \delta(u) d u$.

In the discrete setting for the Haar wavelet, we consider a set of points centered at $n$ or a vertex $V_{n, j}$ of size $2^{j}{ }^{16}$ Assuming that the inner product $\left\langle f, f^{\prime}\right\rangle$ is defined as $\sum_{v \in V} f(v) f^{\prime}(v)$, the $m=0$ scattering coefficients sum the values of $f$ in each $V_{J, n}$, that is,

$$
S_{J} f\left(n, 2^{J-j_{1}}\right)=\left\langle f, 1_{V_{J, n}}\right\rangle .
$$

The order $m=1$ scattering coefficients are the sums of the absolute values of the orthogonal Haar wavelet coefficients,

$$
S_{J} f\left(n, 2^{J-j_{1}}\right)=\sum_{\substack{p \\ V_{j_{1}, p} \subset V_{J, n}}}\left|\left\langle f, \psi_{j_{1}, p}\right\rangle\right| .
$$

The $m=2$ or second ordered scattering coefficients are found by applying the second orthogonal Haar wavelet transform to the first ordered scattering coefficients. In general, the order $m$ coefficients are found by applying the $m$ orthogonal Haar wavelet transform to the $m-1$ scattering coefficients.

\section{SHEARLET EXTRACTED ROBUST FEATURES}

In this section, we introduce a method to extract robust features, which are called Shearlet Extracted Robust Features (SERF) and can be seen as a refinement of the SURF construction. The first step of our approach consists in finding local extrema in the shearlet domain to detect the points of interest. The shearlet transform, defined in Sec. 2, is essentially implemented by combining specific directional filtering obtained by the Fourierbased construction with the application of a Laplacian Pyramid (LP). ${ }^{17}$ Since the directional filtering is applied by convolution, which is a commutative operation, we apply the directional filtering to the image first and then use an efficient implementation of the LP based on the use of integral images. This efficient implementation can be done non-iteratively so that any filter regardless of size can be applied equally fast. In fact, it can be applied in parallel.

The use of the LP allows us to detect points of interest in a manner that is essentially scale invariant by recording the scale of the location of the extreme point and incorporating this scale with regard to the descriptor. The directional filtering in this application enhances edge features in the image so that many keypoint locations can be located at or near subtle edges that would otherwise be undetected.

\subsection{Location of keypoints}

Given a point $x \in \mathbb{R}^{2}$ in the image $f$, we define $G_{x_{1}, x_{2}}\left(x, \sigma_{j}\right)=\frac{\partial^{2}}{\partial x_{1} \partial x_{2}} g\left(\sigma_{j}\right) * f(x)$ where $g\left(\sigma_{j}\right)$ is a Gaussian function with spread $\sigma_{j}$ dependent on a scale $j$. Similarly, we define $G_{x_{2}, x_{1}}\left(x, \sigma_{j}\right)=\frac{\partial^{2}}{\partial x_{2} \partial x_{1}} g\left(\sigma_{j}\right) * f(x)$, $G_{x_{1}, x_{1}}\left(x, \sigma_{j}\right)=\frac{\partial^{2}}{\partial x_{1}^{2}} g\left(\sigma_{j}\right) * f(x)$, and $G_{x_{2}, x_{2}}\left(x, \sigma_{j}\right)=\frac{\partial^{2}}{\partial x_{2}^{2}} g\left(\sigma_{j}\right) * f(x)$. An established method of finding the extrema is to use the determinant of the Hessian

$$
H\left(x, \sigma_{j}\right)=\left(\begin{array}{ll}
G_{x_{1}, x_{1}}\left(x, \sigma_{j}\right) & G_{x_{1}, x_{2}}\left(x, \sigma_{j}\right) \\
G_{x_{2}, x_{1}}\left(x, \sigma_{j}\right) & G_{x_{2}, x_{2}}\left(x, \sigma_{j}\right)
\end{array}\right) ;+
$$

yet we prefer, for computational efficiency, to approximate the second derivatives of these Gaussians by box car filters. ${ }^{18}$ 
A standard non-maximum suppression routine is then applied in $3 \times 3 \times 3$ neighborhoods to localize and reduce possible keypoint locations where the third dimension corresponds to scale. The maxima of the approximate Hessian matrix are also interpolated in scale and image space. ${ }^{18,19}$ Locating these interest points to sub-pixel / sub-scale accuracy in this manner is especially important at higher levels in the decomposition.

\subsection{SERF descriptors}

For the descriptor to be rotation invariant, we identify each keypoint location's prevailing orientation and extract local samples corresponding to that orientation. The major orientation is found by calculating the responses of the Haar-wavelet in the $x$ and $y$ directions in a circular neighborhood of radius $6 \sigma_{j}$ for the scale $\sigma_{j}$ found for the keypoint. The largest sum of all the moduli of the $x$ and $y$ responses within a sliding orientation window, whose support is $\pi / 3$, is designated as the prevailing orientation.

The samples are extracted from a window of size $20 \sigma_{j}$. Figure 2 illustrates examples of the locations of such found keypoints. The squares around these points indicate the local coordinate system and their sizes approximately correspond to the scaled window.
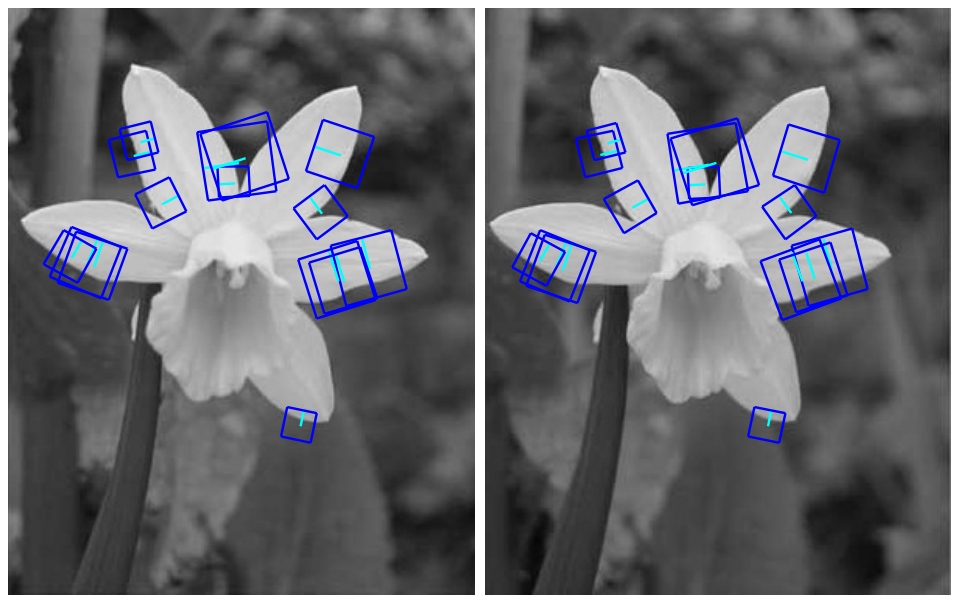

Figure 2. The above images, which differ by a slight angle of capture, show some of the corresponding feature points found by the shearlet-based routine. Overlaid on top of these points is the square descriptor window at different scales used to obtain the scattering feature vectors.

The windowed region is divided into $4 \times 4$ regions and for each sub-region, we calculate the scattering transform values in the $x$ and $y$ directions up to order 2. These values are assembled as a vector of length 6 so that each region is assigned a descriptor vector of length 96 .

For each descriptor in the first image the most similar descriptor in the second image is found using the standard Mahalanobis distance. The matches are kept only if the distances are below a threshold value. This process provides an initial set of correspondences between the paired images and can be used with various transformation and warping estimation routines to aid the registration process.

Figure 3 shows examples of how well the SERF descriptors can match corresponding points in images. For a comparison, we show the results of the matched correspondences from the closely related SURF descriptors.

\section{RESULTS}

In order to investigate the performance of the proposed method, preliminary experiments were performed on two-dimensional image slices extracted from MRI image stacks. These images were obtained from Ref.s 20 and 21. 

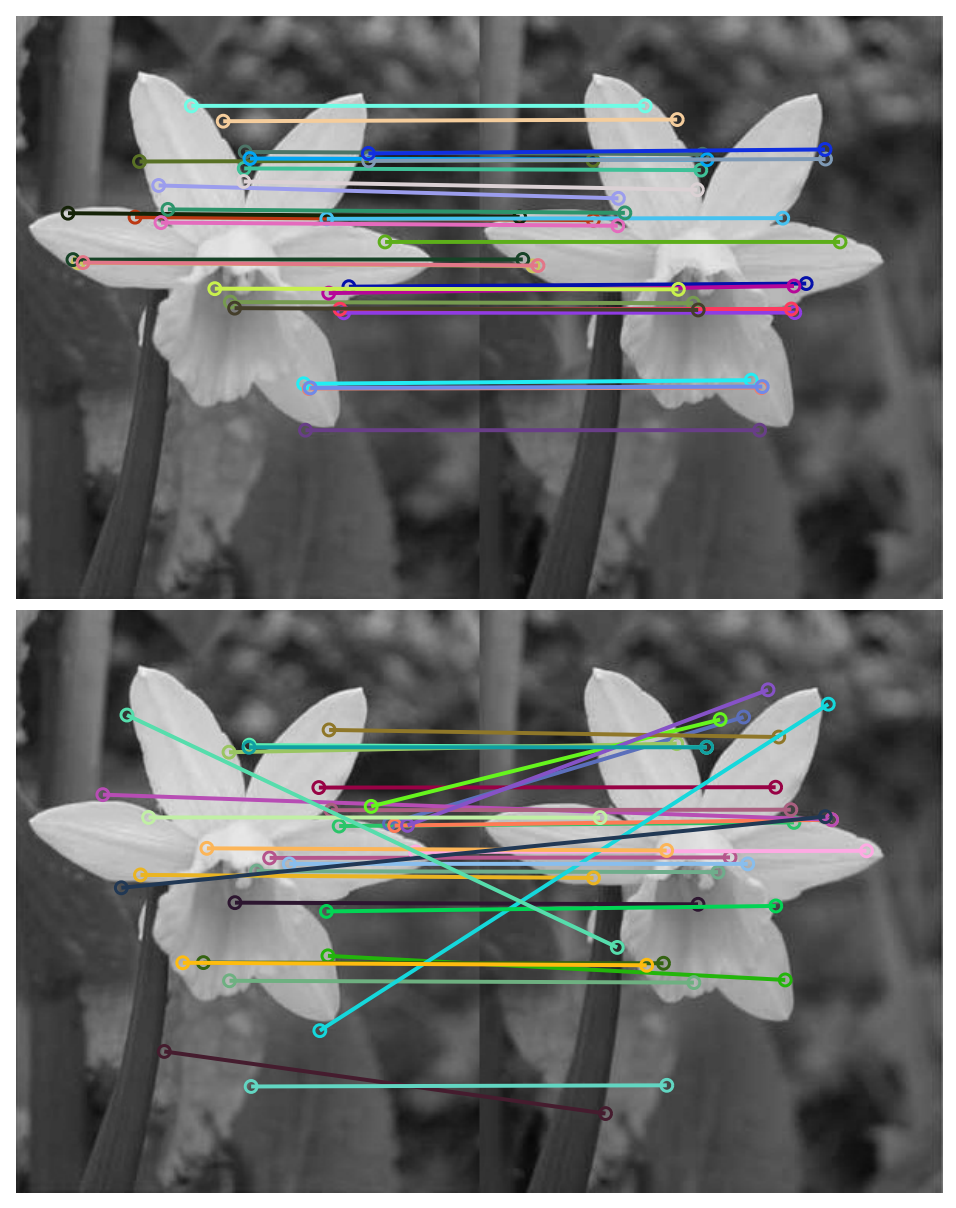

Figure 3. The image on top shows corresponding feature points found using the shearlet transform. The image on the bottom shows corresponding feature points found using SURF (Laplacian Pyramid).

Given two sets of corresponding keypoints from SERF between the test image and the target image to be registered to, a transformation can be identified. Since there are possible mismatches, we used the standard RANSAC algorithm ${ }^{22}$ in the experiments to identify the transformation. Results are shown in Figures 4 and 5.

To demonstrate the ability to register using a warping transformation, we implemented a nonrigid registration using free-form deformations ${ }^{23}$ with the keypoints provided using SERF. The result is shown in Figure 6.

\section{CONCLUSION}

In this work, we have introduced a family of robust and invariant image features based on the scattering transform formed around keypoints, which were found using the shearlet transform. Collectively these points and their descriptors are referred to as Shearlet Extracted Robust Features (SERF).

Using multiple examples, these features were shown to be essentially invariant with respect to rigid transformations and illumination, therefore aiding in challenging registration problems. In fact, these features have been shown to correlate between a sensed and a reference image more accurately than similar features such as SURF. By utilizing RANSAC and free-form deformations, we have demonstrated the effectiveness of these features for tasks of rigid and nonrigid registration on various test images.

In the future, we will further explore the applicability of this approach by incorporating additional feature elements, such as directed graphs, and by extending the method into higher dimensions. 


\section{REFERENCES}

1. L. G. Brown, "A survey of image registration techniques," ACM Comput. Surv. 24, pp. 325-376, Dec. 1992.

2. B. Fischer and J. Modersitzki, "Ill-posed medicinean introduction to image registration," Inverse Problems 24(3), p. 034008, 2008.

3. A. A. Goshtasby, 2-D and 3-D Image Registration: For Medical, Remote Sensing, and Industrial Applications, Wiley-Interscience, 2005.

4. J. Modersitzki, Numerical Methods for Image Registration, Oxford University Press, 2004.

5. O. Scherzer, Mathematical Models for Registration and Applications to Medical Imaging, Mathematics in Industry, Springer, Berlin, 2006.

6. B. Zitov and J. Flusser, "Image registration methods: a survey," Image and Vision Computing 21, pp. 977$1000,2003$.

7. D. Lowe, "Object recognition from local scale-invariant features," in Computer Vision, 1999. The Proceedings of the Seventh IEEE International Conference on, 2, pp. 1150-1157 vol.2, 1999.

8. K. Mikolajczyk and C. Schmid, "A performance evaluation of local descriptors," Pattern Analysis and Machine Intelligence, IEEE Transactions on 27, pp. 1615-1630, Oct 2005.

9. H. Bay, A. Ess, T. Tuytelaars, and L. Van Gool, "Speeded-up robust features (surf)," Comput. Vis. Image Underst. 110, pp. 346-359, June 2008.

10. G. Kutyniok and D. Labate, Shearlets: Multiscale Analysis for Multivariate Data, Springer, 2012.

11. D. Labate, W. Lim, G. Kutyniok, and G. Weiss, "Sparse multidimensional representation using shearlets," SPIE Proc. 5914, SPIE, Bellingham, pp. 254-262, 2005.

12. E. J. Candès and D. L. Donoho, "New tight frames of curvelets and optimal representations of objects with piecewise $C^{2}$ singularities.," Commun. Pure Appl. Anal. 57(2), pp. 219-266, 2004.

13. K. Guo and D. Labate, "The construction of smooth Parseval frames of shearlets.," Math. Model. Nat. Phenom. 8(1), pp. 82-105, 2013.

14. K. Guo and D. Labate, "Optimally sparse multidimensional representation using shearlets," SIAM J. Math. Analysis 39(1), pp. 298-318, 2007.

15. J. Bruna and S. Mallat, "Invariant scattering convolution networks," Pattern Analysis and Machine Intelligence, IEEE Transactions on 35(8), pp. 1872-1886, 2013.

16. X. Chen, X. Cheng, and S. Mallat, "Unsupervised deep haar scattering on graphs," in Advances in Neural Information Processing Systems, pp. 1709-1717, 2014.

17. G. R. Easley, D. Labate, and W. Lim, "Sparse directional image representations using the discrete shearlet transform," Appl. Comput. Harmon. Anal. 25, pp. 25-46, 2008.

18. H. Bay, T. Tuytelaars, and L. Van Gool, "Surf: Speeded up robust features," in Computer vision-ECCV 2006, pp. 404-417, Springer, 2006.

19. M. Brown and D. G. Lowe, "Invariant features from interest point groups.," in BMVC, (1), 2002.

20. B. B. Avants, N. J. Tustison, G. Song, and J. C. Gee, "Ants: Open-source tools for normalization and neuroanatomy," 2010.

21. D.-J. Kroon, "B-spline grid, image and point based registration," MATLAB Central File Exchange, 2011.

22. M. A. Fischler and R. C. Bolles, "Random sample consensus: a paradigm for model fitting with applications to image analysis and automated cartography," Communications of the ACM 24(6), pp. 381-395, 1981.

23. D. Rueckert, L. I. Sonoda, C. Hayes, D. L. Hill, M. O. Leach, and D. J. Hawkes, "Nonrigid registration using free-form deformations: application to breast MR images," Medical Imaging, IEEE Transactions on 18(8), pp. 712-721, 1999. 


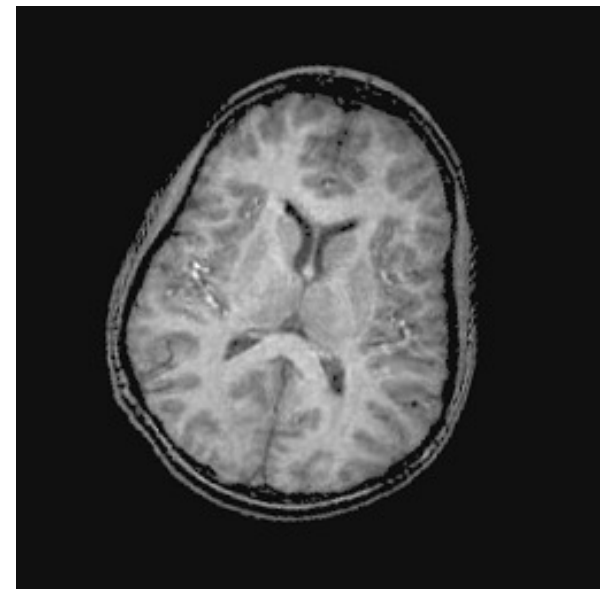

(a)

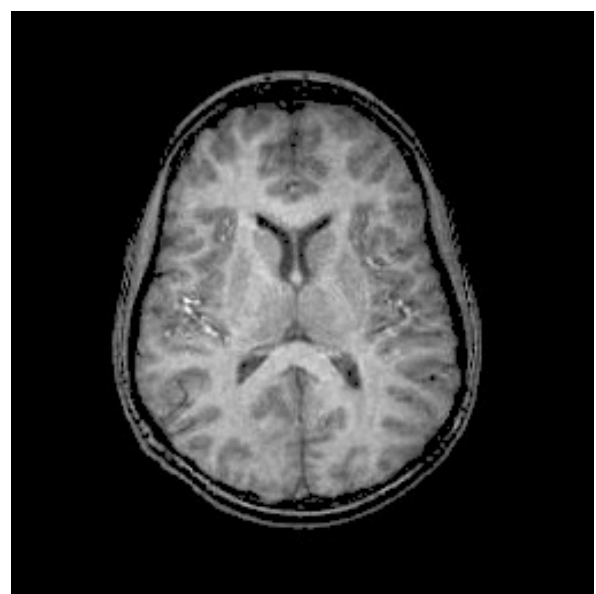

(b)

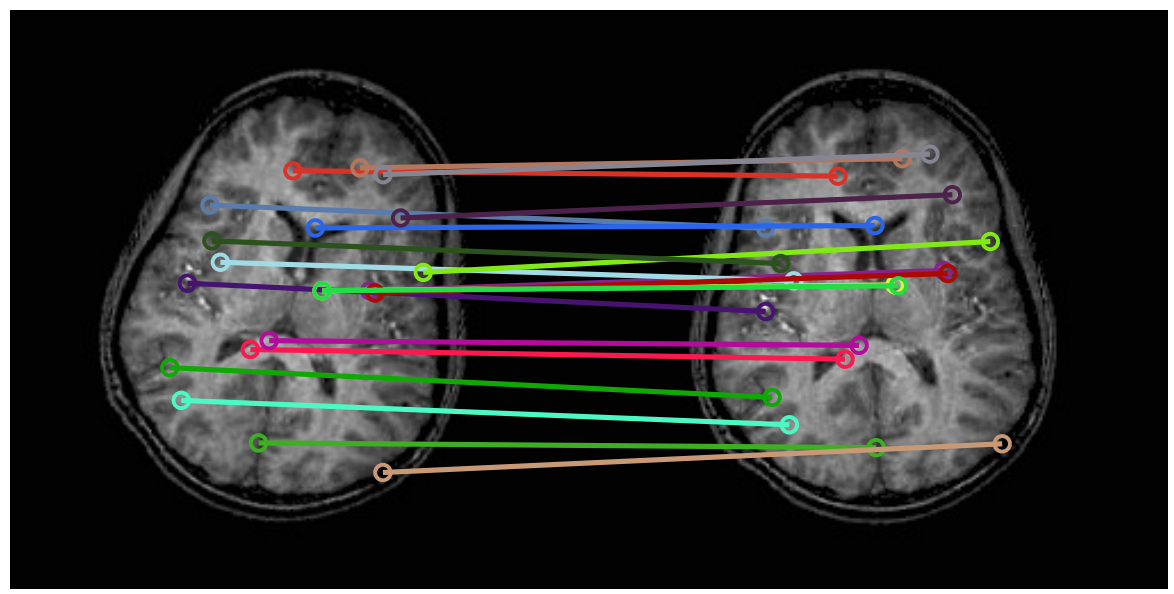

(c)

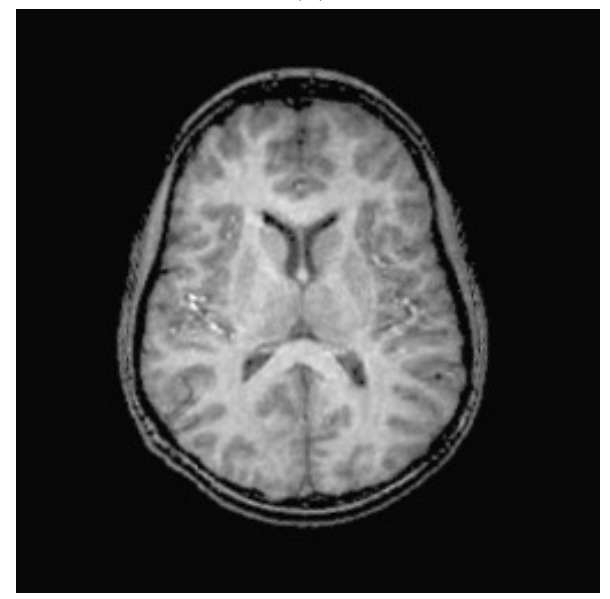

(d)

Figure 4. (a) Test image. (b) Target image to be register to. (c) Matching points found using SERF. (d) Registered image. 


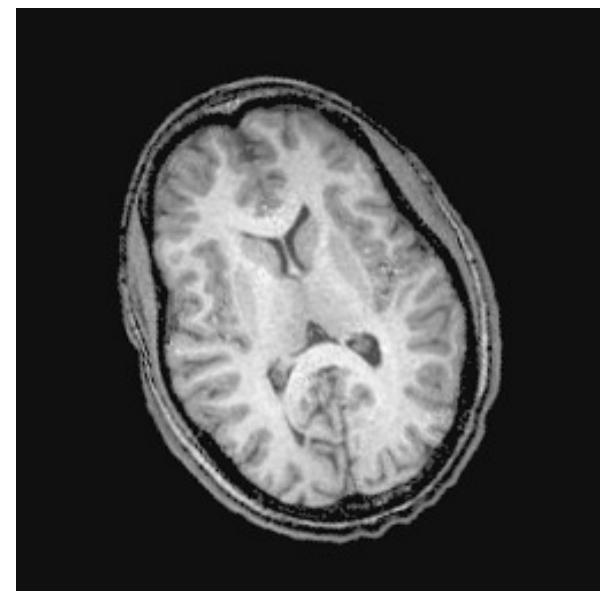

(a)

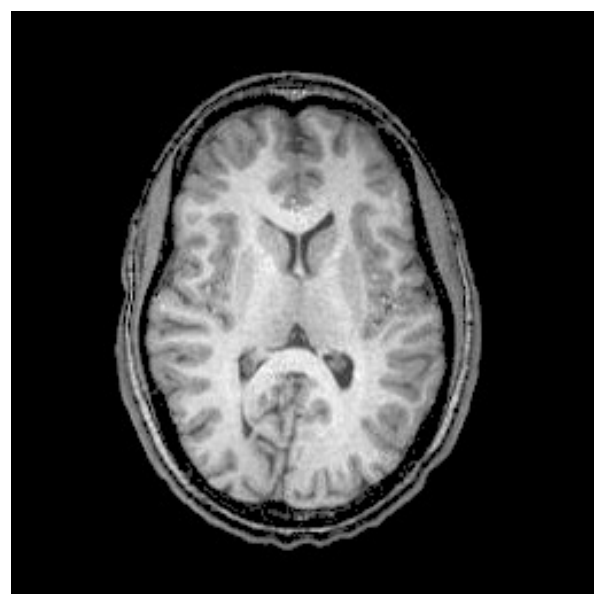

(b)

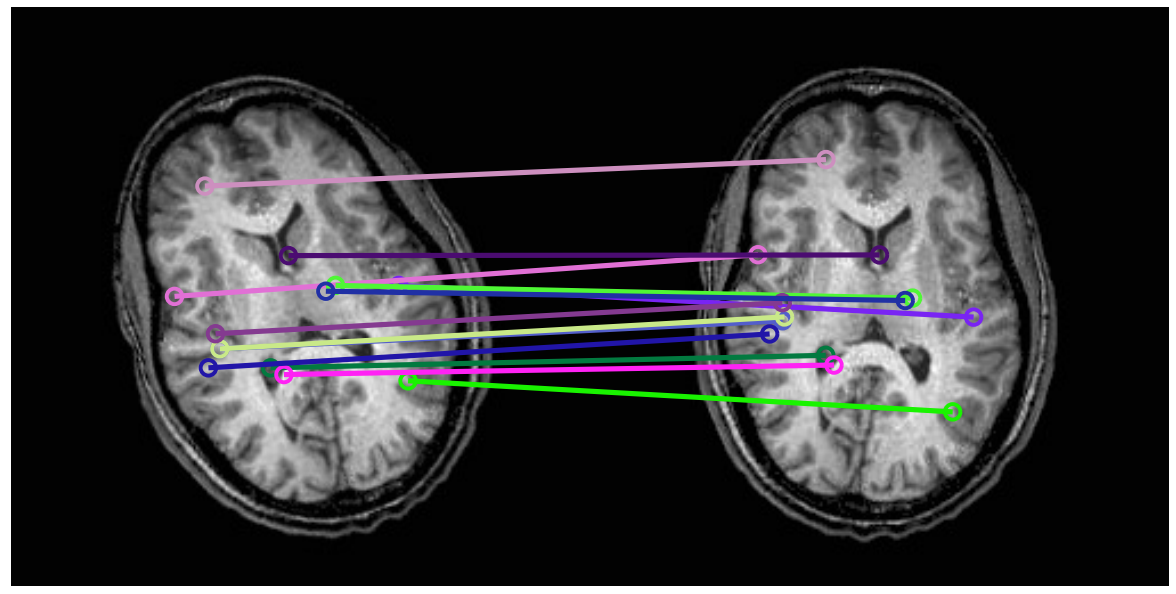

(c)

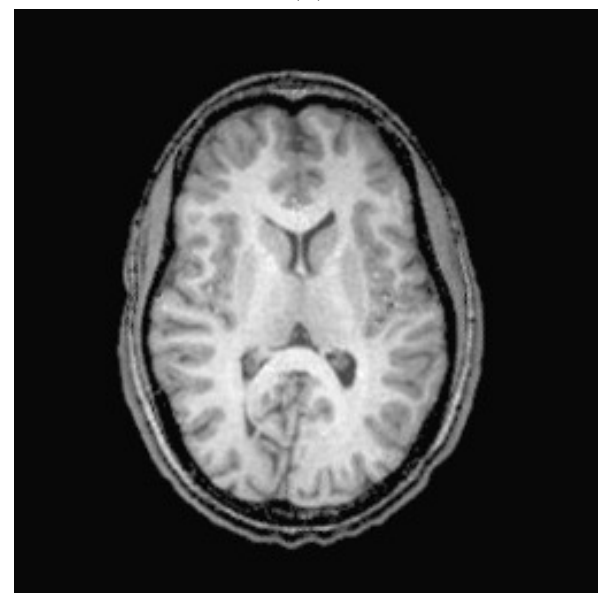

(d)

Figure 5. (a) Test image. (b) Target image to be register to. (c) Matching points found using SERF. (d) Registered image. 


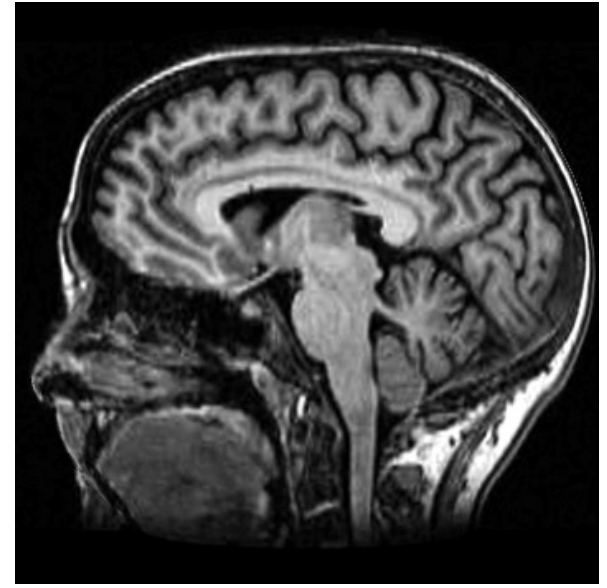

(a)

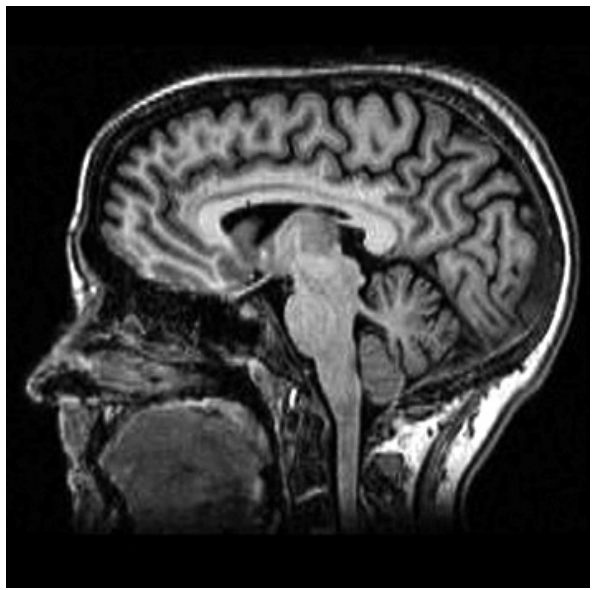

(b)

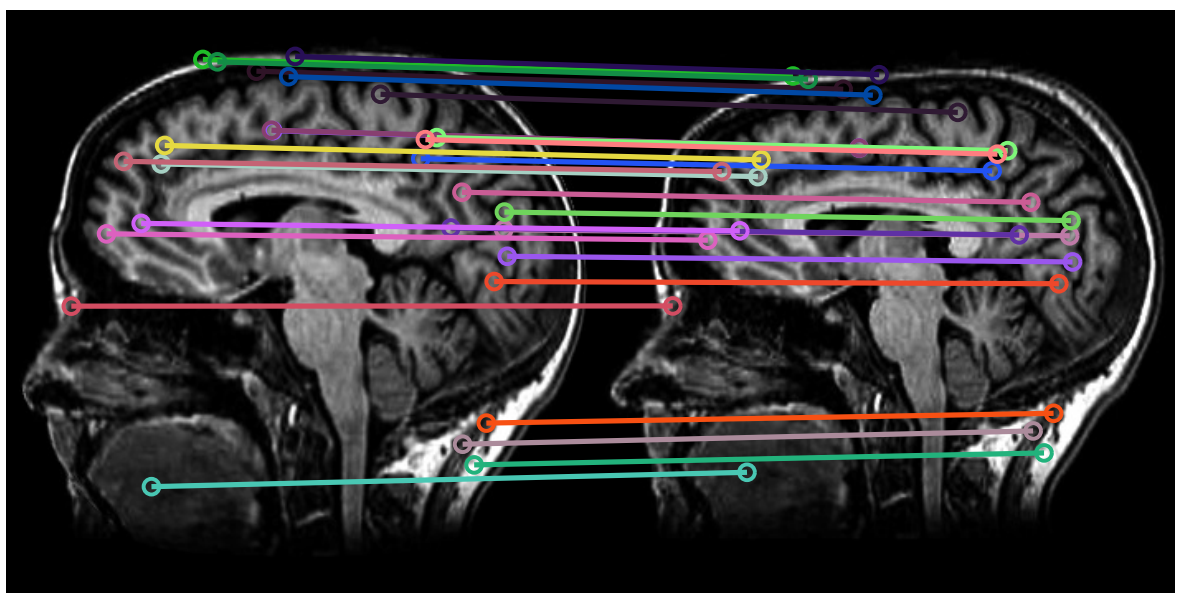

(c)

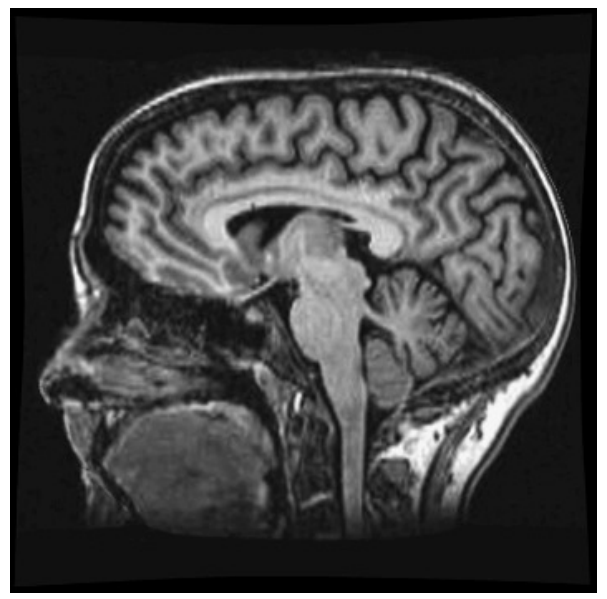

(d)

Figure 6. (a) Test image. (b) Target image to be register to. (c) Matching points found using SERF. (d) Registered image. 